\title{
Unnarmicins A and C, New Antibacterial Depsipeptides Produced by Marine Bacterium Photobacterium sp. MBIC06485
}

\author{
Naoya Oku, Kazutaka Kawabata, Kyoko Adachi, Atsuko Katsuta, Yoshikazu Shizuri
}

Received: September 12, 2007 / Accepted: December 19, 2007

(C) Japan Antibiotics Research Association

\begin{abstract}
Two new antibiotic depsipeptides, unnarmicins C (1) and A (2), were isolated from the fermentation broth of a marine bacterium, Photobacterium sp. strain MBIC06485. The structure of $\mathbf{1}$ was established by spectroscopic studies and chiral analyses of its chemical degradation/conversion products, and that of $\mathbf{2}$ by comparing its NMR, MS, and CD data with those of $\mathbf{1}$. Both compounds selectively inhibited the growth of two strains belonging to the genus Pseudovibrio, one of the most prevalent genera in the marine environments within the class Alphaproteobacteria.
\end{abstract}

Keywords unnarmicin, antibacterial, Photobacterium, marine bacterium

\section{Introduction}

Gram-negative bacteria, especially those belonging to Alphaproteobacteria or Gammaproteobacteria are among the most abundant in the marine environment [1]. However, most studies on metabolites from marine bacteria have been made on Actinomycetes, largely as a reflection of historically proven success with their terrestrial counterparts [2 5]. To exploit the greater potential of marine microbial sources for biomedical applications, we have focused our research interest on unexploited/underexploited taxa for their bioactive metabolites. The results include discovery of cytotoxins from two strains belonging to Thermoactinomycetaceae genera [6 8] and from the Gammaproteobacterium,

K. Adachi (Corresponding author), N. Oku, K. Kawabata, A. Katsuta, Y. Shizuri: Marine Biotechnology Institute Co. Ltd., 3-75-1 Heita, Kamaishi, Iwate 026-0001, Japan,

E-mail: kyoko.adachi@mbio.jp
Pelagiobacter variabilis [9], antibiotic fatty acid derivatives from the Gammaproteobacteria, Pseudoalteromonas sp. [10] and Vibrio gazogenes [11], anti-cyanobacterial peptides from the Alphaproteobacterium, Sphingomonas sp. [12, 13], hydroxamate siderophores from Pseudoalteromonas sp. [14] and the Bacteroidetes, Tenacibaculum sp. [15], and a xanthine oxidase inhibitor from the Alphaproteobacterium, Agrobacterium aurantiacum [16].

In our antibacterial screening of marine bacterial fermentation products against several strains of cosmopolitan marine bacteria, a fermentation extract of an isolate identified as a new Photobacterium (Gammaproteobacteria) showed selective growth inhibition against two strains of Pseudovibrio, one of the most common Alphaproteobacterial genera frequently isolated from the marine environments. This strain is closely related to Photobacterium leiognathi ATCC $25521^{\mathrm{T}}$ (95\% identity based on the 16S rRNA gene sequence), a light organ symbiont for the Leiognathid fish and several other invertebrates [17]. The active components responsible for this unique antibacterial profile were found to be a series of new depsipeptides which were designated as unnarmicins. The taxonomy of the producing organism and fermentation, isolation, and elucidation of the structure study of unnarmicins C (1) and A (2) (Fig. 1) are described below.

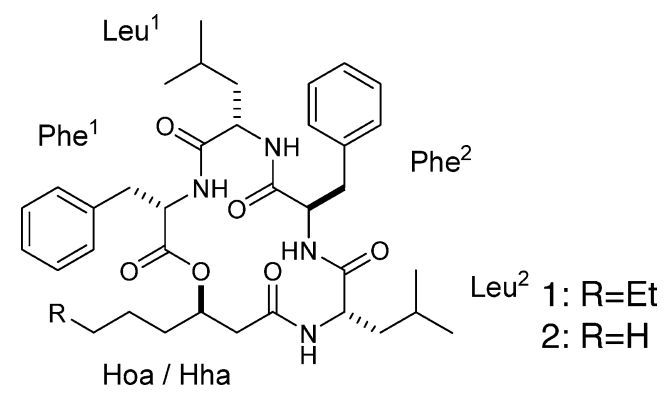

Fig. 1 Structures of unnarmicins C (1) and A (2). 


\section{Results and Discussion}

\section{Taxonomy of Strain MBIC06485}

Cells were facultatively anaerobic, motile, polar-flagellated, Gram-negative bacilli with a size of $2.8 \sim 3.1 \mu \mathrm{m}$ in length and $0.6 \sim 0.7 \mu \mathrm{m}$ in width. Spores were not formed. Colonies grown on Marine Agar 2216 were circular, flattened with a wavy surface, white in the center and translucent at the growing edge. The optimal temperature range for growth is $25 \sim 30^{\circ} \mathrm{C}$. The $\mathrm{pH}$ range for growth was 5 10, optimally $6 \sim 9$. $\mathrm{NaCl}$ was required for growth in the range of $1.0 \sim 7.0 \%$. Tests for enzymatic activities were positive for urease, oxidase, and catalase, but negative for $\beta$-galactosidase. Nitrate was reduced. DNA and starch were hydrolyzed but not esculine. Utilization of citric acid was positive, but hydrogen sulfide and indole production were negative. Acid was produced from D-glucose, Dfructose, maltose, lactose, glycerin, but not from Larabinose, D-xylose, D-mannose, D-galactose, sucrose, trehalose, D-sorbitol, D-mannitol, inositol, or starch. The major isoprenoid quinone was ubiquinone-8. The $\mathrm{G}+\mathrm{C}$ content of DNA was $51.7 \%$. A homology search of the $16 \mathrm{~S}$ rRNA gene sequence of this strain by the BLAST program resulted in the closest type strain being Photobacterium leiognathi ATCC25521 ${ }^{\mathrm{T}} \quad$ (GenBank/EMBL/DDBJ accession No. X74686, 95\% identity). These morphological and physiological characteristics enabled the strain to be identified as a member of the genus Photobacterium.

\section{Isolation}

The EtOAc extract $(780 \mathrm{mg})$ of the fermentation broth $(10$ liters) was chromatographed on silica gel eluted with $\mathrm{CHCl}_{3} / \mathrm{MeOH}(20: 1)$ to give a crude anti-Pseudovibrio fraction $(50 \mathrm{mg})$. Purification of this fraction by reversed phase HPLC eluted with $60 \%$ aq $\mathrm{MeCN}$ yielded $\mathbf{1}$ and $\mathbf{2}$ as white amorphous solids.

\section{Structure Elucidation}

The molecular formula of $\mathbf{1}$ was established as $\mathrm{C}_{38} \mathrm{H}_{54} \mathrm{~N}_{4} \mathrm{O}_{6}$ $\left(\mathrm{m} / \mathrm{z} 663.4138[\mathrm{M}+\mathrm{H}]^{+}\right.$, calcd for $\mathrm{C}_{38} \mathrm{H}_{55} \mathrm{~N}_{4} \mathrm{O}_{6}, 663.4122$, $\Delta 1.6 \mathrm{mmu}$ ) by an HRFAB-MS analysis. The ${ }^{1} \mathrm{H}$ - and ${ }^{13} \mathrm{C}$ NMR and DEPT spectra in DMSO- $d_{6}$ were typical of those for peptides, as showing four each of amide linkages (Table 1: $\left.\delta_{\mathrm{H}} 7.35 \sim 8.94 ; \delta_{\mathrm{C}} 167.73 \sim 173.55\right)$ and methines in the mid-magnetic regions $\left(\delta_{\mathrm{H}} 4.03 \sim 4.52 ; \delta_{\mathrm{C}} 50.09 \sim 55.88\right)$. Interpretation of the COSY and TOCSY spectra gave 5 spin systems, of which four were accounted for by a pair of Leu (Leu ${ }^{1}: \delta_{\mathrm{H}} 8.20,4.03,1.35,1.14,0.97,0.67,0.60$; Leu $^{2}$ : $\left.\delta_{\mathrm{H}} 7.35,4.52,1.43,1.33,1.47,0.87,0.83\right)$ and a pair of Phe

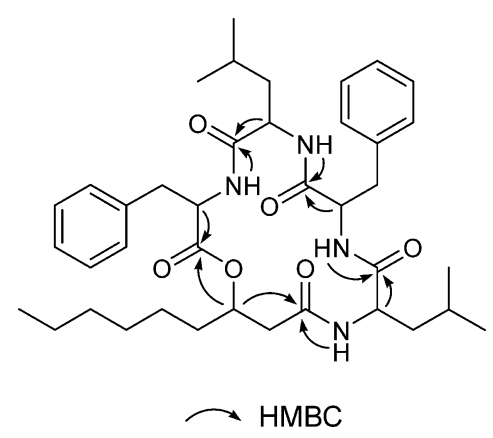

Fig. 2 Key HMBC correlations for 1.

$\left(\mathrm{Phe}^{1}: \delta_{\mathrm{H}} 7.82,7.29,7.26,7.20,4.36,3.21,3.08\right.$; $\mathrm{Phe}^{2}:$ $\left.\delta_{\mathrm{H}} 8.94,7.27,7.26,7.20,4.31,2.91\right)$ residues and the remaining one by a 3 -hydroxyoctanoyl moiety $(=\mathrm{Hoa}, \delta$ $2.56,2.14,4.99,1.51,1.43,1.06,1.16,1.21,0.83)$. HMBC correlations (Fig. 2) from the amide protons to adjacent amide carbons, $\delta_{\mathrm{H}} / \delta_{\mathrm{C}} 7.82\left(\mathrm{Phe}^{1}\right) / 171.95\left(\mathrm{Leu}^{1}\right), 8.20$ $\left(\mathrm{Leu}^{1}\right) / 170.99\left(\mathrm{Phe}^{2}\right), 8.94\left(\mathrm{Phe}^{2}\right) / 173.55\left(\mathrm{Leu}^{2}\right)$, revealed an alternating alignment of Phe and Leu, whereas HMBC correlations $\delta_{\mathrm{H}} / \delta_{\mathrm{C}} \quad 7.35 / 167.73, \quad 4.99 / 167.73, \quad$ and 4.99/169.89 were respective evidence for $N$ - and $C$-terminal attachment of Hoa to $\mathrm{Leu}^{2}$ and Phe ${ }^{1}$, thereby completing the peptide lactone structure for $\mathbf{1}$.

A chiral analysis of the amino acid components in $\mathbf{1}$ after derivatizing its acid-hydrolysate with L-FDAA ( $=(5$-fluoro2,4-dinitrophenyl)-L-alaninamide, Marfey's reagent) revealed the presence of L-Leu and an enantiomeric pair of Phe (Scheme 1). To specify the stereochemistry of each Phe residue, 1 was reduced by $\mathrm{LiBH}_{4}$ and resulting linear product 3 was subjected to the same analytical protocol. Since a new peak (Rt 25.1 minutes, attributable to Lphenylalaninol:L-Phl) appeared in exchange for the disappearance of the L-Phe peak (Rt 23.2 minutes), L- and D-stereochemistry were assigned for $\mathrm{Phe}^{1}$ and $\mathrm{Phe}^{2}$, respectively.

The absolute configuration of $\mathrm{C} 3$ in Hoa was determined by applying the modified Mosher method to 3. The plus and minus $\Delta \delta$ values consistently resided towards the $\omega$ - and carboxyl termini, respectively (Fig. 3), enabling the $R$-configuration to be concluded for this stereocenter.

The HRFAB-MS spectrum for $\mathbf{2}$ gave a molecular ion at $m / z 635.3812\left([\mathrm{M}+\mathrm{H}]^{+}\right.$, calcd. for $\mathrm{C}_{36} \mathrm{H}_{51} \mathrm{~N}_{4} \mathrm{O}_{6}, 635.3809$, $+\Delta 0.3 \mathrm{mmu}$ ), revealing the molecular formula of 2 to be two methylene units smaller than that of $\mathbf{1}$. An analysis of the NMR data for $\mathbf{2}$ immediately disclosed that this difference was due to replacement of the Hoa unit in $\mathbf{1}$ with a 3-hydroxyhexanoyl (Hha) group. The stereochemistry of $\mathbf{2}$ was concluded to be the same as that of $\mathbf{1}$ based on a CD spectral comparison, in which $\mathbf{1}$ and $\mathbf{2}$ showed almost identical spectra (Fig. 4). 
Table 1 NMR data for unnarmicins C (1) and A (2)

\begin{tabular}{|c|c|c|c|c|c|c|c|}
\hline \multicolumn{4}{|c|}{1} & \multicolumn{4}{|c|}{2} \\
\hline Position & $\delta_{\mathrm{C}}(\mathrm{m})$ & & $\delta_{\mathrm{H}} J(\mathrm{~Hz})$ & Position & $\delta_{\mathrm{C}}(\mathrm{m})$ & & $\delta_{\mathrm{H}} J(\mathrm{~Hz})$ \\
\hline $\mathrm{Phe}^{1}$ & & & & $\mathrm{Phe}^{1}$ & & & \\
\hline $\mathrm{NH}$ & & 7.82 & $1 \mathrm{H}, \mathrm{d}, 8.8$ & $\mathrm{NH}$ & & 7.80 & $1 \mathrm{H}, \mathrm{d}, 8.8$ \\
\hline$\alpha$ & 54.67 (d) & 4.36 & $1 \mathrm{H}, \mathrm{dt}, 5.6,8.9$ & $\alpha$ & 54.65 (d) & 4.36 & $1 \mathrm{H}, \mathrm{dt}, 5.6,8.9$ \\
\hline \multirow[t]{2}{*}{$\beta$} & $37.10(t)$ & 3.21 & $1 \mathrm{H}, \mathrm{dd}, 9.1,14.2$ & $\beta$ & $37.13(t)$ & 3.19 & $1 \mathrm{H}, \mathrm{dd}, 9.5,14.2$ \\
\hline & & 3.08 & $1 \mathrm{H}, \mathrm{dd}, 5.6,14.2$ & & & 3.08 & $1 \mathrm{H}, \mathrm{dd}, 5.6,14.2$ \\
\hline 1 & 137.57 (s) & & & 1 & 137.52 (s) & & \\
\hline $2 / 6$ & $128.95(d)$ & 7.29 & $2 \mathrm{H}, \mathrm{m}$ & $2 / 6$ & $128.95(d)$ & 7.28 & $2 \mathrm{H}, \mathrm{m}$ \\
\hline $3 / 5$ & $127.82(d)$ & 7.26 & $1 \mathrm{H}, \mathrm{m}$ & $3 / 5$ & $127.83(d)$ & 7.25 & $1 \mathrm{H}, \mathrm{m}$ \\
\hline 4 & $126.18(d)$ & 7.20 & $1 \mathrm{H}, \mathrm{m}$ & 4 & $126.20(d)$ & 7.19 & $1 \mathrm{H}, \mathrm{m}$ \\
\hline $\mathrm{CO}$ & 169.89 (s) & & & $\mathrm{CO}$ & 169.85 (s) & & \\
\hline Leu $^{1}$ & & & & $\mathrm{Leu}^{1}$ & & & \\
\hline $\mathrm{NH}$ & & 8.20 & $1 \mathrm{H}, \mathrm{d}, 9.0$ & $\mathrm{NH}$ & & 8.20 & $1 \mathrm{H}, \mathrm{d}, 9.0$ \\
\hline$\alpha$ & 50.79 (d) & 4.03 & $1 \mathrm{H}, \mathrm{ddd}, 3.3,9.0,12.4$ & $\alpha$ & $50.85(d)$ & 3.98 & $1 \mathrm{H}, \mathrm{m}$ \\
\hline \multirow[t]{2}{*}{$\beta$} & $39.34(t)$ & 1.35 & $1 \mathrm{H}, \mathrm{m}$ & $\beta$ & $39.37(t)$ & 1.33 & $1 \mathrm{H}, \mathrm{m}$ \\
\hline & & 1.14 & $1 \mathrm{H}, \mathrm{m}$ & & & 1.12 & $1 \mathrm{H}, \mathrm{m}$ \\
\hline$\gamma$ & $23.44(d)$ & 0.97 & $1 \mathrm{H}, \mathrm{m}$ & $\gamma$ & $23.44(d)$ & 0.97 & $1 \mathrm{H}, \mathrm{m}$ \\
\hline$\delta$ & $23.13(q)$ & 0.67 & $3 \mathrm{H}, \mathrm{d}, 6.7$ & $\delta$ & $23.13(q)$ & 0.67 & $3 \mathrm{H}, \mathrm{d}, 6.7$ \\
\hline$\delta^{\prime}$ & $20.58(q)$ & 0.60 & $3 \mathrm{H}, \mathrm{d}, 6.4$ & $\delta^{\prime}$ & $20.62(q)$ & 0.59 & $3 \mathrm{H}, \mathrm{d}, 6.7$ \\
\hline $\mathrm{CO}$ & 171.95 (s) & & & $\mathrm{CO}$ & 171.97 (s) & & \\
\hline $\mathrm{Phe}^{2}$ & & & & $\mathrm{Phe}^{2}$ & & & \\
\hline $\mathrm{NH}$ & & 8.94 & $1 \mathrm{H}, \mathrm{d}, 5.8$ & $\mathrm{NH}$ & & 8.92 & $1 \mathrm{H}, \mathrm{d}, 5.8$ \\
\hline$\alpha$ & $55.88(d)$ & 4.31 & $1 \mathrm{H}, \mathrm{dt}, 5.8,7.7$ & $\alpha$ & 55.87 (d) & 4.30 & $1 \mathrm{H}, \mathrm{dt}, 6.0,7.8$ \\
\hline$\beta$ & $35.94(t)$ & 2.91 & $2 \mathrm{H}, \mathrm{d}, 7.7$ & $\beta$ & $35.94(t)$ & 2.91 & $2 \mathrm{H}, \mathrm{d}, 7.7$ \\
\hline 1 & 136.92 (s) & & & 1 & 136.89 (s) & & \\
\hline $2 / 6$ & $129.06(d)$ & 7.27 & $2 \mathrm{H}, \mathrm{m}$ & $2 / 6$ & $129.06(d)$ & 7.27 & $2 \mathrm{H}, \mathrm{m}$ \\
\hline $3 / 5$ & $128.05(d)$ & 7.26 & $1 \mathrm{H}, \mathrm{m}$ & $3 / 5$ & $128.06(d)$ & 7.25 & $1 \mathrm{H}, \mathrm{m}$ \\
\hline 4 & 126.07 (d) & 7.20 & $1 \mathrm{H}, \mathrm{m}$ & 4 & $126.08(d)$ & 7.19 & $1 \mathrm{H}, \mathrm{m}$ \\
\hline $\mathrm{CO}$ & 170.99 (s) & & & $\mathrm{CO}$ & 171.00 (s) & & \\
\hline $\mathrm{Leu}^{2}$ & & & & $\mathrm{Leu}^{2}$ & & & \\
\hline $\mathrm{NH}$ & & 7.35 & $1 \mathrm{H}, \mathrm{d}, 8.6$ & $\mathrm{NH}$ & & 7.35 & $1 \mathrm{H}, \mathrm{d}, 8.6$ \\
\hline$\alpha$ & 50.09 (d) & 4.52 & $1 \mathrm{H}, \mathrm{dt}, 6.3,9.0$ & $\alpha$ & 50.10 (d) & 4.52 & $1 \mathrm{H}, \mathrm{dt}, 6.3,9.0$ \\
\hline \multirow[t]{2}{*}{$\beta$} & $40.78(t)$ & 1.43 & $1 \mathrm{H}, \mathrm{m}$ & $\beta$ & $40.77(t)$ & 1.43 & $1 \mathrm{H}, \mathrm{m}$ \\
\hline & & 1.33 & $1 \mathrm{H}, \mathrm{m}$ & & & 1.33 & $1 \mathrm{H}, \mathrm{m}$ \\
\hline$\gamma$ & $24.18(d)$ & 1.47 & $1 \mathrm{H}, \mathrm{m}$ & $\gamma$ & $24.18(d)$ & 1.47 & $1 \mathrm{H}, \mathrm{m}$ \\
\hline$\delta$ & $22.66(q)$ & 0.87 & $3 \mathrm{H}, \mathrm{d}, 6.5$ & $\delta$ & $22.67(q)$ & 0.88 & $3 \mathrm{H}, \mathrm{d}, 6.3$ \\
\hline$\delta^{\prime}$ & $21.86(q)$ & 0.83 & $3 \mathrm{H}, \mathrm{t}, 7.5$ & $\delta^{\prime}$ & $21.85(q)$ & 0.84 & $3 \mathrm{H}, \mathrm{d}, 6.7$ \\
\hline $\mathrm{CO}$ & 173.55 (s) & & & $\mathrm{CO}$ & 173.52 (s) & & \\
\hline Hoa & & & & Hha & & & \\
\hline 1 & 167.73 (s) & & & 1 & 167.73 (s) & & \\
\hline \multirow[t]{2}{*}{2} & $39.50(t)$ & 2.56 & $1 \mathrm{H}, \mathrm{dd}, 4.2,13.5$ & 2 & $39.45(t)$ & 2.57 & $1 \mathrm{H}, \mathrm{dd}, 4.2,13.5$ \\
\hline & & 2.14 & $1 \mathrm{H}, \mathrm{dd}, 4.2,13.5$ & & & 2.14 & $1 \mathrm{H}, \mathrm{dd}, 4.2,13.5$ \\
\hline 3 & $71.55(d)$ & 4.99 & $1 \mathrm{H}, \mathrm{m}$ & 3 & $71.23(d)$ & 5.01 & $1 \mathrm{H}, \mathrm{m}$ \\
\hline \multirow[t]{2}{*}{4} & $32.00(t)$ & 1.51 & $1 \mathrm{H}, \mathrm{m}$ & 4 & $34.12(t)$ & 1.51 & $1 \mathrm{H}, \mathrm{m}$ \\
\hline & & 1.43 & $1 \mathrm{H}, \mathrm{m}$ & & & 1.43 & $1 \mathrm{H}, \mathrm{m}$ \\
\hline 5 & $24.66(t)$ & 1.06 & $2 \mathrm{H}, \mathrm{m}$ & 5 & $18.19(t)$ & 1.08 & $2 \mathrm{H}, \mathrm{m}$ \\
\hline 6 & $30.84(t)$ & 1.16 & $2 \mathrm{H}, \mathrm{m}$ & 6 & $13.59(q)$ & 0.79 & $3 \mathrm{H}, \mathrm{d}, 6.7$ \\
\hline 7 & $21.91(t)$ & 1.21 & $2 \mathrm{H}, \mathrm{m}$ & & & & \\
\hline 8 & $13.80(q)$ & 0.83 & $3 \mathrm{H}, \mathrm{t}, 7.5$ & & & & \\
\hline
\end{tabular}


The antibacterial activity of $\mathbf{1}$ and $\mathbf{2}$ against two Grampositive bacteria (Firmicutes) and seven halophilic Gram negative bacteria belonging to Bacteroidetes, Alphaproteobacteria, and Gammaproteobacteria were evaluated by a plate diffusion assay. As summarized in Table 2, the peptides specifically inhibited the growth of two Pseudovibrio strains (Alphaproteobacteria). Such unique action suggests potential application of the peptides, in combination with korormicin [10] which is a selective antibiotic against halophilic Gammaproteobacteria, as an

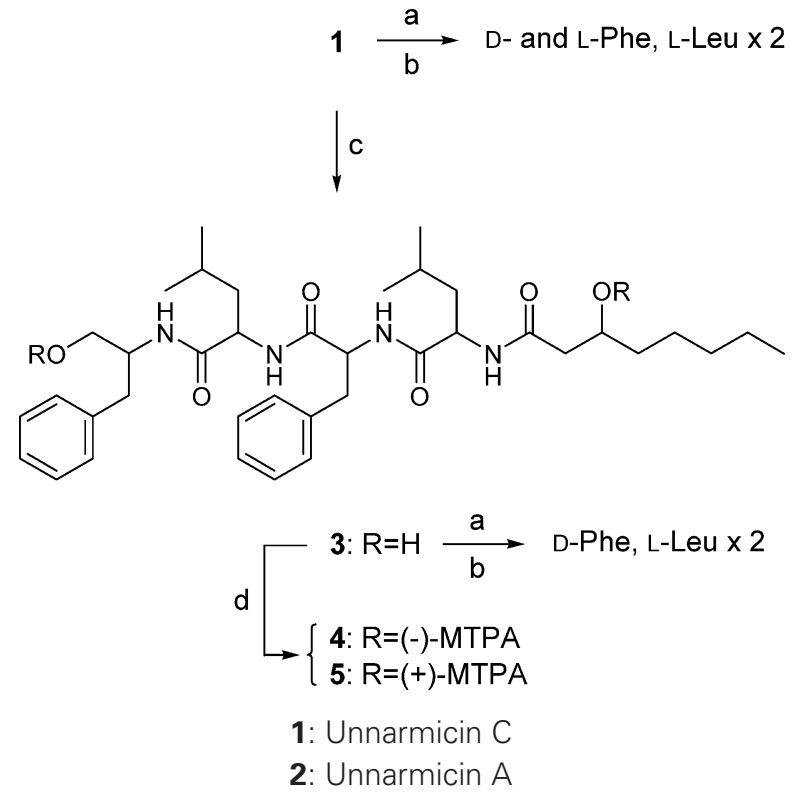

Scheme 1

Reagents and conditions: (a) $6 \mathrm{~N} \mathrm{HCl}, 110^{\circ} \mathrm{C}, 18$ hours; (b) LFDAA, $0.1 \mathrm{~N} \mathrm{NaHCO}_{3} / \mathrm{Me}_{2} \mathrm{CO}(2: 1), 70^{\circ} \mathrm{C}$; (c) $\mathrm{LiBH}_{4}, \mathrm{THF}^{\circ} 0^{\circ} \mathrm{C}$ to rt, 2 hours; (d) (-)- or (+)-MTPACl, pyridine, rt, 2 hours. ideal selection additive to media to suppress fast-growing bacteria that often dominate on solidified media and hamper the isolation/purification of slow-growing colonies. The few precedents of secondary metabolites identified from Photobacterium are all pteridines [18, 19], thus the

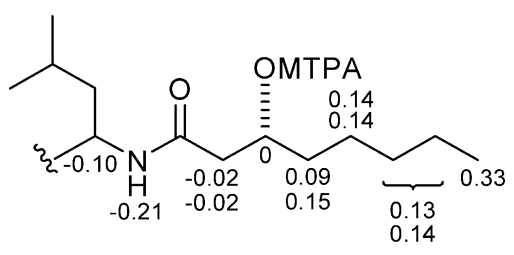

$\Delta \delta=\delta_{\mathrm{H}}(\mathbf{4})-\delta_{\mathrm{H}}(\mathbf{5})$

Fig. 3 Distribution of $\delta \Delta$ values calculated for the Hoa unit.

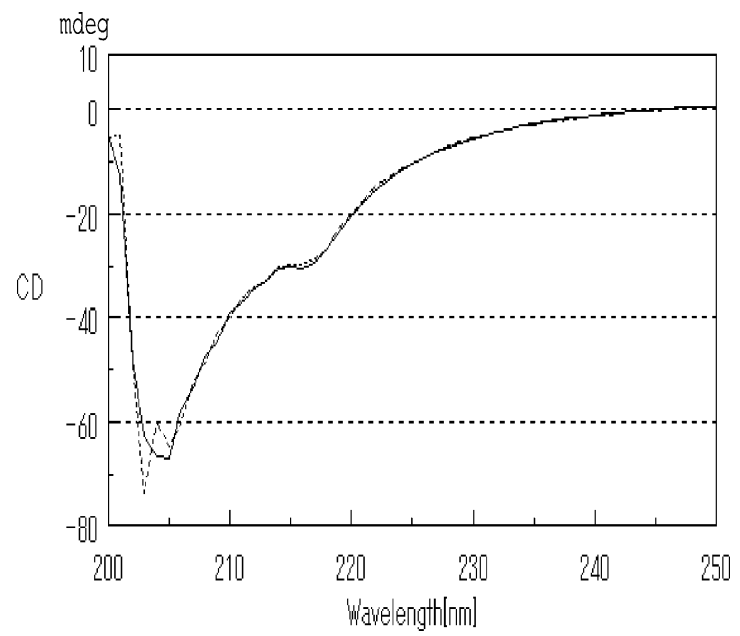

Fig. 4 Superimposed CD spectra for $\mathbf{1}$ (solid line) and 2 (dotted line) in $\mathrm{MeOH}$. Concn.: $80 \mu \mathrm{M}$

Table 2 Antibacterial activities of $\mathbf{1}$ and $\mathbf{2}$ in the plate diffusion assay

\begin{tabular}{|c|c|c|c|c|c|c|c|c|}
\hline \multirow{2}{*}{ Tested strains } & \multicolumn{2}{|c|}{1} & \multicolumn{2}{|c|}{2} & \multirow{2}{*}{$\begin{array}{l}P^{a} \\
2.6\end{array}$} & \multirow{2}{*}{$\begin{array}{l}\mathrm{PB}^{b} \\
2.6\end{array}$} & \multirow{2}{*}{$\begin{array}{l}\mathrm{CP}^{c} \\
2.6\end{array}$} & \multirow{2}{*}{$\begin{array}{r}\text { Koro } \\
2.6\end{array}$} \\
\hline & $13^{e}$ & 2.6 & 13 & 2.6 & & & & \\
\hline Staphylococcus aureus IFO12732 & & & & & $13^{f}$ & 24 & 14 & \\
\hline Bacillus subtilis IFO3134 & & & & & 9 & 24 & 19 & \\
\hline \multicolumn{9}{|l|}{ Cytophaga marinoflava IFO14170 } \\
\hline Pseudovibrio denitrificans JCM12308 & 7 & 7 & 18 & 10 & 15 & & & \\
\hline Pseudovibrio sp. MBIC3368 & 8 & 7 & 12 & 9 & 31 & & & \\
\hline Agrobacterium kieliense IAM12618 & & & & & 14 & & 16 & \\
\hline Caulobacter halobacteroides NCIMB2022 & & & & & 13 & & & \\
\hline Salinivibrio costicola ATCC33508 & & & & & & 9 & & 25 \\
\hline Vibrio harveyi IFO15634 & & & & & & & 13 & 20 \\
\hline
\end{tabular}

${ }^{a}$ Penicillin G. ${ }^{b}$ Polymyxin B. ${ }^{c}$ Chloramphenicol. ${ }^{d}$ Korormicin. ${ }^{e}$ Amount of compound (mg) applied to the paper disk. ${ }^{f}$ Diameter of inhibition zone (mm). 
discovery of the unnarmicins demonstrates a rather richer biosynthetic potential for this genus. Details of the biological properties of $\mathbf{1}$ and $\mathbf{2}$ will be published elsewhere.

\section{Experimental}

\section{General Experimental Procedures}

Optical rotations were measured with a Horiba SEPA-300 high sensitivity polarimeter. UV and IR spectra were obtained with Beckman DU640 and Jasco FT-IR 7000 spectrophotometers, respectively, whereas CD spectra with Jasco J-725 spectropolarimeter in $\mathrm{MeOH}$. NMR spectra were recorded with Varian UNITY INOVA 750 or 500 spectrometers, referencing chemical shifts to the residual solvent signals at $\delta_{\mathrm{H}} 2.50$ and $\delta_{\mathrm{C}} 39.5 \mathrm{ppm}$ in DMSO- $d_{6}$ and at $\delta_{\mathrm{H}} 7.25$ and $\delta_{\mathrm{C}} 77.0 \mathrm{ppm}$ in $\mathrm{CDCl}_{3}$. Mass spectra were measured with a Jeol JMS-SX102A instrument for HRFAB-MS and with a ThermoFinigan LCQ Advantage instrument for ESI-MS.

\section{Producing Microorganisms and Fermentation}

Photobacterium sp. strain MBIC06485 was isolated from coastal seawater collected off Onna Beach, Okinawa, Japan. A subculture of this isolate has been deposited at National Institute of Technology and Evaluation (Kisarazu, Chiba, Japan) with accession number NITE P-62.

To produce the unnarmicins, a 2-ml seed culture of strain MBIC06485 grown in Marine Broth 2216 (Difco) was transferred to $300 \mathrm{ml}$ of the same medium in a 1-liter baffled Erlenmeyer flask. The flask was shaken at $100 \mathrm{rpm}$ and $30^{\circ} \mathrm{C}$ for 4 days before harvesting the fermentation products.

\section{Isolation of 1 and 2}

The fermentation broth was centrifuged at $6000 \mathrm{rpm}$ for 20 minutes to remove the bacterial cells. The resulting supernatant (10 liters) was extracted with EtOAc and the extract was dried over anhydrous $\mathrm{Na}_{2} \mathrm{SO}_{4}$, after which the solvent was removed in vacuo to give an oily solid $(780 \mathrm{mg})$. This material was fractionated by $\mathrm{SiO}_{2}$ chromatography using isocratic elution with $\mathrm{CHCl}_{3}: \mathrm{MeOH}$ $(20: 1)$, and the combined antibacterial fraction $(50 \mathrm{mg})$ against Pseudovibrio sp. MBIC3368 was purified by reversed-phase HPLC (Shiseido CAPCELL PAK C18 UG120 column, $20 \times 250 \mathrm{~mm}, 60 \%$ aq $\mathrm{MeCN}$ as a solvent, detection at $210 \mathrm{~nm})$ to yield $\mathbf{1}(6.0 \mathrm{mg})$ and $\mathbf{2}(3.7 \mathrm{mg})$.

Unnarmicin C (1)

White amorphous solid; $[\alpha]_{\mathrm{D}}^{30} 67^{\circ}(c 0.084, \mathrm{MeOH})$; UV
(MeOH) $\lambda_{\max } 258.5 \mathrm{~nm}(\varepsilon 400) ; \mathrm{CD}(\mathrm{MeOH}) \lambda_{\mathrm{ext}} 216 \mathrm{~nm}$ $(\Delta \varepsilon-12, \mathrm{sh}), 205$ (-65); IR (KBr) $v_{\max } 3297,2956,2927$, 2854, 1747, 1717, 1670, 1654, 2359, 1691, 1631, 1557, 1542, 1523, $1508 \mathrm{~cm}^{-1}$; HRFAB-MS $\mathrm{m} / \mathrm{z} 663.4138$ $[\mathrm{M}+\mathrm{H}]^{+}$, calcd. for $\mathrm{C}_{38} \mathrm{H}_{55} \mathrm{~N}_{4} \mathrm{O}_{6}, 663.4122 .{ }^{1} \mathrm{H}-$ and ${ }^{13} \mathrm{C}-$ NMR data, see Table 1.

Unnarmicin A (2)

White amorphous solid; $[\alpha]_{\mathrm{D}}^{30} 70^{\circ}(c 0.19, \mathrm{MeOH})$; UV $(\mathrm{MeOH}) \lambda_{\max } 258.5 \mathrm{~nm}(\varepsilon 410) ; \mathrm{CD}(\mathrm{MeOH}) \lambda_{\mathrm{ext}} 216 \mathrm{~nm}$ $(\Delta \varepsilon-12, \mathrm{sh}), 205$ (-67); IR (KBr) $v_{\max } 3297,2956,2927$, 2854, 1747, 1717, 1670, 1654, 2359, 1691, 1631, 1557, 1542, 1523, $1508 \mathrm{~cm}^{-1}$; HRFAB-MS $\mathrm{m} / z \quad 635.3812$ $[\mathrm{M}+\mathrm{H}]^{+}$, calcd. for $\mathrm{C}_{36} \mathrm{H}_{51} \mathrm{~N}_{4} \mathrm{O}_{6}$ for $635.3809 .{ }^{1} \mathrm{H}$ - and ${ }^{13} \mathrm{C}$ NMR data, see Table 1.

\section{Antibacterial Assay}

The antibacterial activities of $\mathbf{1}$ and $\mathbf{2}$ was evaluated by the paper disk method. A liquid culture of each test strain ( $4.0 \mathrm{ml}$, cultured for 24 hours) was smeared with a sterile cotton-tipped swab onto a Marine Agar 2216 (Difco) or Nutrient Agar (Difco) medium using a sterile cotton-tipped swab. Methanolic solutions of the peptides were prepared at concentrations of 1.0 and $0.2 \mathrm{mg} / \mathrm{ml}$, and a $13 \mu \mathrm{l}$-aliquot from each solution was applied to a $6 \mathrm{~mm}$-paper disk. The disks were air-dried for $10 \sim 20$ minutes and then transferred to the test plates, which were incubated at $30^{\circ} \mathrm{C}$ for 24 hours. The antibacterial potency of each peptide was evaluated by measuring the diameter of the generated inhibitory zones.

\section{Chiral Analysis of the Component Amino Acids in 1}

A $50-\mu \mathrm{g}$ portion of 1 was hydrolyzed in constant-boiling hydrochloric acid $(6 \mathrm{~N}, 200 \mu \mathrm{l})$ at $105^{\circ} \mathrm{C}$ for 19 hours in a $\mathrm{N}_{2}$ atmosphere. The dried acid hydrolysate, which was obtained after removing hydrochloric acid under a stream of $\mathrm{N}_{2}$, was dissolved in $0.1 \mathrm{~N}$ aq $\mathrm{NaHCO}_{3}(100 \mu \mathrm{l})$ and reacted with L-FDAA $\left(0.1 \%\right.$ solution in $\left.\mathrm{Me}_{2} \mathrm{CO}, 50 \mu \mathrm{l}\right)$ at $50^{\circ} \mathrm{C}$ for 30 minutes. The reaction was quenched with $0.2 \mathrm{~N}$ hydrochloric acid $(50 \mu \mathrm{l})$ and the mixture was diluted with $50 \%$ aq MeCN containing $50 \mathrm{mM} \mathrm{NH}_{4} \mathrm{OAc}(100 \mu \mathrm{l})$ to give an analyte solution. A $10-\mu 1$ aliquot of this solution was analyzed by reversed-phase HPLC on a Cosmosil AR-II column $(4.6 \times 150 \mathrm{~mm})$ with the elution program of $15 \%$ MeCN - $50 \mathrm{mM} \mathrm{NH}_{4} \mathrm{OAc}$ for 5 minutes $(1.0 \mathrm{ml} /$ minute flow rate), $0.67 \% /$ minute concentration gradient of $\mathrm{MeCN}$ to $45 \%$, and then an $11 \%$ /minute gradient to $100 \%$, and the same concentration held for 5 minutes. Analytical standards were prepared from $0.1 \mathrm{mg}$ each of authentic amino acids with the same derivatization procedure as that already described. Retention times (minute): L-Leu, 21.3: 
L-Phe, 23.2; D-Leu, 28.5; D-Phe, 29.5; acid hydrolysate of 1, 21.3, 23.2, and 29.5.

\section{Stereochemical Analysis of the Phe Residues in 1}

To the peptide $(1.5 \mathrm{mg})$ in a sealed pear-shaped flask was added a solution of $\mathrm{LiBH}_{4}$ in THF $(2.0 \mathrm{M}, 200 \mu \mathrm{l})$ and the reaction mixture was stirred first at $0^{\circ} \mathrm{C}$ for 5 minutes and then at $\mathrm{rt}$ for 2 hours. The mixture was diluted with $\mathrm{Et}_{2} \mathrm{O}$ $(0.5 \mathrm{ml})$ and the reaction was quenched by stirring with a saturated aqueous $\mathrm{NH}_{4} \mathrm{Cl}$ solution $(0.2 \mathrm{ml})$ until the generation of bubbles had ceased. The mixture was again diluted with $\mathrm{Et}_{2} \mathrm{O}(4.0 \mathrm{ml})$ and dried over anhydrous $\mathrm{MgSO}_{4}$ for 10 minutes. The $\mathrm{Et}_{2} \mathrm{O}$ solution and the washings were passed through a plug of glasswool layered on cotton in a Pasteur pipette. The residue in the flask was dissolved in $\mathrm{H}_{2} \mathrm{O}$ and loaded onto ODS $(2 \mathrm{ml})$, which was successively eluted with $\mathrm{H}_{2} \mathrm{O}$ and $\mathrm{MeOH}$. The $\mathrm{MeOH}$ eluate was combined with the ethereal filtrate and purified by HPLC on a Cosmosil AR-II column $(1 \times 25 \mathrm{~cm})$ with a gradient elution with aq $\mathrm{MeCN}(30 \sim 60 \%)$ to give linear reduction product $3(0.2 \mathrm{mg})$ : ESI-MS $m / z 667.4[\mathrm{M}+\mathrm{H}]^{+}$; ${ }^{1} \mathrm{H}-\mathrm{NMR}\left(750 \mathrm{MHz}, \mathrm{CDCl}_{3}\right) \delta 3.76$ (brs, $1 \mathrm{H}, \mathrm{CH}_{2} \mathrm{OH}, \mathrm{Phl}$ ), $3.74\left(\mathrm{~d}, J=12.2 \mathrm{~Hz}, 1 \mathrm{H},-\mathrm{CH}_{2} \mathrm{OH}, \mathrm{Phl}\right), 3.42(\mathrm{~m}, 1 \mathrm{H},-$ $\left.\mathrm{CH}_{2} \mathrm{OH}, \mathrm{Phl}\right), 4.10(\mathrm{~m}, 1 \mathrm{H}, \alpha \mathrm{H}, \mathrm{Phl}), 2.87(\mathrm{~m}, 1 \mathrm{H}, \beta \mathrm{Ha}$, $\mathrm{Phl}$ ), 2.97 (m, 1H, $\beta \mathrm{Hb}, \mathrm{Phl}), 7.25$ (H2/6, Phl), 7.27 (H3/5, Phl), 7.19 (H4, Phl), 6.84 (d, J=12.2 Hz, NH, Phl), 4.08 $\left(\alpha \mathrm{H}\right.$, Leu $\left.^{1}\right), 1.57\left(\beta \mathrm{Ha}\right.$, Leu $\left.^{1}\right), 1.16\left(\beta \mathrm{Hb}\right.$, Leu $\left.^{1}\right), 0.84(\mathrm{~m}$, $\left.1 \mathrm{H}, \gamma \mathrm{H}, \mathrm{Leu}^{1}\right), 0.66\left(\mathrm{~d}, J=6.4 \mathrm{~Hz}, 3 \mathrm{H}, \delta \mathrm{H}, \mathrm{Leu}^{1}\right), 0.71(\mathrm{~d}$, $\left.J=6.7 \mathrm{~Hz}, 3 \mathrm{H}, \delta^{\prime} \mathrm{H}, \mathrm{Leu}^{1}\right), 5.79\left(\mathrm{~d}, J=7.3 \mathrm{~Hz}, \mathrm{~N} H, \mathrm{Leu}^{1}\right)$, $4.20(\mathrm{~m}, 1 \mathrm{H}, \alpha \mathrm{H}, \mathrm{Phe}), 3.00 \sim 3.04$ (m, 1H, $\beta \mathrm{H}, \mathrm{Phe}), 7.17$ (H2/6, Phe), 7.30 (H3/5, Phe), 7.26 (H4, Phe), 7.27 (NH, Phe), $4.47\left(\alpha \mathrm{H}, \mathrm{Leu}^{2}\right), 1.55\left(\beta \mathrm{Ha}, \mathrm{Leu}^{2}\right), 1.75\left(\beta \mathrm{Hb}, \mathrm{Leu}^{2}\right)$, $1.63\left(\gamma \mathrm{H}, \mathrm{Leu}^{2}\right), 0.92\left(\delta \mathrm{H}, \mathrm{Leu}^{2}\right), 0.93\left(\delta^{\prime} \mathrm{H}, \mathrm{Leu}^{2}\right), 6.76(\mathrm{~d}$, $\left.J=8.7 \mathrm{~Hz}, \mathrm{~N} H, \mathrm{Leu}^{2}\right), 2.31$ (dd, $J=14.2,6.2 \mathrm{~Hz}, \mathrm{H} 2 \mathrm{a}, \mathrm{Hoc}$ ), 2.50 (dd, $J=3.9,14.3 \mathrm{~Hz}, \mathrm{H} 2 \mathrm{~b}, \mathrm{Hoc}$ ), 3.94 (H3, Hoc), 3.86 (brs, OH, Hoc), 1.61 (H4a, Hoc), 1.70 (m, H4b, Hoc), 1.40 (H5, Hoc), 1.49 (H5, Hoc), 1.35 (H6, Hoc), 1.32 1.35 (H7, Hoc), 0.90 (H8, Hoc); ${ }^{13} \mathrm{C}-\mathrm{NMR}\left(125 \mathrm{MHz}, \mathrm{CDCl}_{3}\right) \delta$ 62.8 ( $\left.-\mathrm{CH}_{2} \mathrm{OH}, \mathrm{Phl}\right), 52.9(\alpha \mathrm{C}, \mathrm{Phl}), 36.8$ ( $\left.\beta \mathrm{C}, \mathrm{Phl}\right), 138.5$ (C1, Phl), 129.4 (C2/6, Phl), 128.5 (C3/5, Phl), 126.5 (C4, Phl), $171.2\left(C \mathrm{O}, \mathrm{Leu}^{1}\right), 53.2\left(\alpha \mathrm{C}, \mathrm{Leu}^{1}\right), 40.3\left(\beta \mathrm{C}, \mathrm{Leu}^{1}\right)$, $24.5\left(\gamma \mathrm{C}\right.$, Leu $\left.^{1}\right), 21.5\left(\delta \mathrm{C}\right.$, Leu$\left.^{1}\right), 23.3\left(\delta^{\prime} \mathrm{C}, \mathrm{Leu}^{1}\right), 172.2$ (CO, Phe), 57.5 ( $\alpha \mathrm{C}, \mathrm{Phl}), 37.3$ ( $\beta \mathrm{C}, \mathrm{Phl}), 135.9(\mathrm{Cl}, \mathrm{Phl})$, 129.1 (C2/6, Phl), 128.9 (C3/5, Phl), 127.3 (C4, Phl), 173.6 $\left(C \mathrm{O}, \mathrm{Leu}^{2}\right), 50.9\left(\alpha \mathrm{C}, \mathrm{Leu}^{2}\right), 38.8\left(\beta \mathrm{C}, \mathrm{Leu}^{2}\right), 25.0(\gamma \mathrm{C}$, $\left.\mathrm{Leu}^{2}\right), 22.5\left(\delta \mathrm{C}, \mathrm{Leu}^{1}\right), 23.0\left(\delta^{\prime} \mathrm{C}, \mathrm{Leu}^{1}\right), 173.2(\mathrm{C} 1, \mathrm{Hoa})$, 43.1 (C2, Hoa), 69.3 (C3, Hoa), 36.6 (C4, Hoa), 25.8 (C5, Hoa), 22.8 (C6, Hoa), 31.9 (C7, Hoa), 14.2 (C8, Hoa). A $10 \mu \mathrm{g}$-portion of $\mathbf{3}$ was hydrolyzed and subjected to the same analytical protocol as described in the former section, resulting in a loss of the L-Phe peak. Retention time (minute): acid-hydrolysate of 3,21.2 and 29.3.

\section{Preparation of Bis-MTPA Esters from Linear Derivative 3}

To each $0.1 \mathrm{mg}$-aliquot of derivative $\mathbf{3}$ in a V-bottom vial was added 10 drops of dry pyridine and $(-)$ - or $(+)$ MTPACl $(9 \mu 1)$. The reaction vial was vigorously stirred at $\mathrm{rt}$ for 30 minutes and then centrifuged at $6000 \mathrm{rpm}$ for 1.5 hours. After removing pyridine and the excess reagent by lyophilization, the residue was purified by reversed-phase HPLC (Cosmosil AR-II column, $1 \times 25 \mathrm{~cm}, 50 \%$ aq $\mathrm{MeCN}$ to $\mathrm{MeCN}$ as the solvent) to give bis-MTPA esters, respectively.

\section{Bis-(-)-MTPA Ester (4)}

${ }^{1} \mathrm{H}-\mathrm{NMR}\left(750 \mathrm{MHz}, \mathrm{CDCl}_{3}\right) \delta 4.47\left(-\mathrm{CH}_{2} \mathrm{OH}, \mathrm{Phl}\right), 4.12$ $(\mathrm{m}, 1 \mathrm{H}, \alpha \mathrm{H}, \mathrm{Phl}), 2.76(\mathrm{~m}, 1 \mathrm{H}, \beta \mathrm{Ha}, \mathrm{Phl}), 2.86(\mathrm{~m}, 1 \mathrm{H}$, $\beta \mathrm{Hb}, \mathrm{Phl}), 6.97$ (br, NH, Phl), $4.30\left(\alpha \mathrm{H}, \mathrm{Leu}^{1}\right), 1.51(\beta \mathrm{H}$, $\left.\mathrm{Leu}^{1}\right), 1.23\left(\gamma \mathrm{H}, \mathrm{Leu}^{1}\right), 0.78\left(\mathrm{~d}, J=6.8 \mathrm{~Hz}, 3 \mathrm{H}, \delta \mathrm{H}, \mathrm{Leu}^{1}\right)$, $0.79\left(\mathrm{~d}, J=6.8 \mathrm{~Hz}, 3 \mathrm{H}, \delta^{\prime} \mathrm{H}, \mathrm{Leu}^{1}\right), 6.30$ (br, NH, Leu ${ }^{1}$ ), $4.54(\alpha \mathrm{H}$, Phe), 2.77 (m, $\beta \mathrm{Ha}, \mathrm{Phe}), 3.21$ (dd, $J=6.9$, $13.6 \mathrm{~Hz}, \beta \mathrm{Hb}$, Phe), 6.76 (br, NH, Phe), $4.11\left(\alpha \mathrm{H}, \mathrm{Leu}^{2}\right)$, $1.47\left(\beta \mathrm{H}, \mathrm{Leu}^{2}\right), 1.18\left(\gamma \mathrm{H}, \mathrm{Leu}^{2}\right), 0.77(\mathrm{~d}, J=6.0 \mathrm{~Hz}, \delta \mathrm{H}$, $\left.\mathrm{Leu}^{2}\right), 0.80\left(\mathrm{~d}, J=6.0 \mathrm{~Hz}, \delta^{\prime} \mathrm{H}, \mathrm{Leu}^{2}\right), 6.07(\mathrm{~d}, J=6.0 \mathrm{~Hz}$, $\mathrm{N} H, \mathrm{Leu}^{2}$ ), 2.44 (dd, $\left.J=15.0,4.0 \mathrm{~Hz}, \mathrm{H} 2 \mathrm{a}, \mathrm{Hoc}\right), 2.50$ (dd, $J=7.8,15.0 \mathrm{~Hz}, \mathrm{H} 2 \mathrm{~b}, \mathrm{Hoc}$ ), 5.50 (m, H3, Hoc), 1.69 (H4a, Hoc), 1.75 (H4b, Hoc), 1.28 1.31 (H5, Hoc), 1.27 1.32 (H6/7, Hoc), 0.85 (t, $J=6.8 \mathrm{~Hz}, \mathrm{H} 8$, Hoc), 3.46, (s, $\mathrm{CH}_{3} \mathrm{O}-$, MTPA) 3.50 (s, $\mathrm{CH}_{3} \mathrm{O}-$, MTPA). Signals for phenyl rings were not assigned.

\section{Bis-(+)-MTPA Ester (5)}

${ }^{1} \mathrm{H}-\mathrm{NMR}\left(750 \mathrm{MHz}, \mathrm{CDCl}_{3}\right) \delta 4.12(\mathrm{dd}, J=3.4,10.6 \mathrm{~Hz}$, $\left.\mathrm{CH}_{2} \mathrm{OH}, \mathrm{Phl}\right), 4.51$ (dd, $J=7.3,10.5 \mathrm{~Hz},-\mathrm{CH}_{2} \mathrm{OH}, \mathrm{Phl}$ ), $4.466(\mathrm{~m}, 1 \mathrm{H}, \alpha \mathrm{H}, \mathrm{Phl}), 2.816$ (m, 1H, $\beta \mathrm{Ha}, \mathrm{Phl}), 2.88$ (dd, $J=7.1,13.9 \mathrm{~Hz}, 1 \mathrm{H}, \beta \mathrm{Hb}, \mathrm{Phl}), 7.04$ (br NH, Phl), 4.31 $\left(\alpha \mathrm{H}, \mathrm{Leu}^{1}\right), 1.52\left(\beta \mathrm{H}, \mathrm{Leu}^{1}\right), 1.16\left(\gamma \mathrm{H}, \mathrm{Leu}^{1}\right), 0.76(\mathrm{~d}$, $\left.J=6.6 \mathrm{~Hz}, 3 \mathrm{H}, \delta / \delta^{\prime} \mathrm{H}, \mathrm{Leu}^{1}\right), 6.08\left(\mathrm{~d}, J=7.8 \mathrm{~Hz}, \mathrm{~N} H, \mathrm{Leu}^{1}\right)$, $4.455(\alpha \mathrm{H}, \mathrm{Phe}), 2.814(\beta \mathrm{Ha}, \mathrm{Phe}), 3.19(\mathrm{dd}, J=7.4$, $13.8 \mathrm{~Hz}, \beta \mathrm{Hb}, \mathrm{Phe}), 6.87$ (br, NH, Phe), 4.21 (dt, $J=7.1$, $\left.7.1 \mathrm{~Hz}, \alpha \mathrm{H}, \mathrm{Leu}^{2}\right), 1.51\left(\beta \mathrm{H}, \mathrm{Leu}^{2}\right), 1.20\left(\gamma \mathrm{H}, \mathrm{Leu}^{2}\right), 0.77$ $\left(\mathrm{d}, J=5.9 \mathrm{~Hz}, \delta \mathrm{H}, \mathrm{Leu}^{2}\right), 0.78\left(\mathrm{~d}, J=5.9 \mathrm{~Hz}, \delta^{\prime} \mathrm{H}, \mathrm{Leu}^{2}\right)$, $6.28\left(\mathrm{~d}, J=6.5 \mathrm{~Hz}, \mathrm{~N} H, \mathrm{Leu}^{2}\right), 2.46(\mathrm{dd}, J=15.2,4.3 \mathrm{~Hz}$, H2a, Hoc), 2.52 (dd, $J=8.3,15.2 \mathrm{~Hz}, \mathrm{H} 2 \mathrm{~b}, \mathrm{Hoc}$ ), 5.50 (m, H3, Hoc), 1.60 (H4a, Hoc), 1.14 1.17 (H5, Hoc), $1.13 \sim 1.19$ (H6/7, Hoc), 0.82 (t, $J=6.9 \mathrm{~Hz}, \mathrm{H} 8, \mathrm{Hoc}$ ), 3.52 (s, $\mathrm{CH}_{3} \mathrm{O}-$, MTPA) 3.50 (s, $\mathrm{CH}_{3} \mathrm{O}-$, MTPA). Signals for phenyl rings were not assigned.

Acknowledgments We thank Dr. Ryuichi Sakai (Kitasato University) for assistance with HRFAB-MS measurements, Mr. 
Satoru Matsuda (MBI) for ESI-MS measurements, and Dr. Hiroaki Kasai (MBI) for the taxonomy. This study was performed as part of the project entitled "Construction of a Genetic Resource Library of Unidentified Microorganisms" supported by New Energy Industrial Technology Development Organization (NEDO).

\section{References}

1. Venter JC, Remington K, Heidelberg JF, Halpen AL, Rusch D, Eisen JA, Wu D, Paulsen I, Nelson KE, Nelson W, Fouts DE, Levy S, Knap AH, Lomas MW, Nealson K, White O, Peterson J, Hoffman J, Parsons R, Baden-Tillson H, Pfannkoch C, Rogers Y-H, Smith HO. Environmental genome shot sequence of the Sargasso Sea. Science 304: 66-74 (2004)

2. Berdy J. Bioactive Microbial Metabolites. A Personal View. J Antibiot 58: 1-26 (2005)

3. Kelecom A. Secondary metabolites from marine microorganisms. An Acad Bras Cienc 74: 151-170 (2002)

4. Jensen PR, Mincer TJ, Williams PG, Fenical W. Marine actinomycete diversity and natural product discovery. Antonie Van Leeuwenhoek 87: 43-48 (2005)

5. Lam KS. Discovery of novel metabolites from marine actinomycetes. Curr Opin Microbiol 9: 245-251 (2006)

6. Matsuo Y, Kanoh K, Imagawa H, Adachi K, Nishizawa M, Shizuri Y. Urukthapelstatin A, a novel cytotoxic substance from marine-derived Mechercharimyces asporophorigenens YM11-542. J Antibiot 60: 256-260 (2007)

7. Matsuo Y, Kanoh K, Yamori T, Kasai H, Katsuta A, Adachi K, Shin-Ya K, Shizuri Y. Urukthapelstatin A, a novel cytotoxic substance from marine-derived Mechercharimyces asporophorigenens YM11-542. J Antibiot 60: 251-255 (2007)

8. Kanoh K, Matsuo Y, Adachi K, Imagawa H, Nishizawa M, Shizuri Y. Mechercharmycins A and B, cytotoxic substances from marine-derived Thermoactinomyces sp. YM3-251. J Antibiot 58: 289-292 (2005)
9. Imamura N, Nishijima M, Takadera T, Adachi K, Sakai M, Sano H. New anticancer antibiotics pelagiomicins, produced by a new marine bacterium Pelagiobacter variabilis. J Antibiot 50: 8-12 (1997)

10. Yoshikawa K, Takadera T, Adachi K, Nishijima M, Sano H. Korormicin, a novel antibiotic specifically active against marine Gram-negative bacteria, produced by a marine bacterium. J Antibiot 50: 949-953 (1997)

11. Imamura N, Adachi K, Sano H. Magnesidin A, a component of marine antibiotic magnesidin, produced by Vibrio gazogenes ATCC29988. J Antibiot 47: 257-261 (1994)

12. Imamura N, Motoike I, Noda M, Adachi K, Konno A, Fukami H. Argimicin A, a novel anti-cyanobacterial compound produced by an algae-lysing bacterium. J Antibiot 53: 1317-1319 (2000)

13. Yamaguchi $\mathrm{T}$, Kobayashi $\mathrm{Y}$, Adachi $\mathrm{K}$, Imamura $\mathrm{N}$. Argimicins B and C, new anti-cyanobacterial compounds produced by Sphingomonas sp. M-17. J Antibiot 56: 655-657 (2003)

14. Kanoh K, Kamino K, Leleo G, Adachi K, Shizuri Y. Pseudoalterobactins A and B, new siderophores excreted by marine bacterium Pseudoalteromonas sp. KP20-4. J Antibiot 56: 871-875 (2003)

15. Jang JH, Kanoh K, Adachi K, Matsuda S, Shizuri Y. Tenacibactins $A \sim D$, hydroxamate siderophores from a marine-derived bacterium, Tenacibaculum sp. A4K-17. J Nat Prod 70: 563-566 (2007)

16. Izumida H, Adachi K, Mihara A, Yasuzawa T, Sano H. Hydroxyakalone, a novel xanthine oxidase inhibitor produced by a marine bacterium, Agrobacterium aurantiacum. J Antibiot 50: 916-918 (1997)

17. Haygood MG. Light organ symbioses in fishes. Crit Rev Microbiol 19: 191-216 (1993)

18. Matsuura S, Odaka M, Sugimoto T, Goto T. The structure of pteridines from Photobacterium. Chem Lett 4: 343-346 (1973)

19. Suzuki A, Goto M. Photolumazines, new naturally occurring inhibitors of riboflavine synthetase. Biochim Biophys Acta 313: 229-234 (1973) 